Research Article

\title{
The Depth of Generalized Full Terms and Generalized Full Hypersubstitutions
}

\author{
Sarawut Phuapong and Sorasak Leeratanavalee \\ Department of Mathematics, Faculty of Science, Chiang Mai University, Chiang Mai 50200, Thailand \\ Correspondence should be addressed to Sorasak Leeratanavalee; sorasak.1@cmu.ac.th
}

Received 7 April 2013; Revised 3 June 2013; Accepted 3 June 2013

Academic Editor: Andrei V. Kelarev

Copyright (c) 2013 S. Phuapong and S. Leeratanavalee. This is an open access article distributed under the Creative Commons Attribution License, which permits unrestricted use, distribution, and reproduction in any medium, provided the original work is properly cited.

\begin{abstract}
We generalize the concept of a full term which was introduced by Denecke and Changphas and the concept of the depth of a hypersubstitution which was introduced by Denecke et al. to the depth of a generalized full term and the depth of a generalized full hypersubstitution and then derive the formular for its depth.
\end{abstract}

\section{Introduction}

Identities are used to classify algebras into collections called varieties. An important activity is to classify all varieties of algebras of a given type $\tau$. Such as for a type $\tau=(2)$, if the binary operation symbol satisfies the associative law, the algebras are semigroups. Hyperidentities are also used to classify varieties into collections called hypervarieties. Its generalization is called $M$-hyperidentities and $M$-solid varieties. The study of hyperidentities is a part of Universal Algebra and also a part of Model Theory of second-order language. The notions of hyperidentities and hypervarieties of a given type $\tau$ without nullary operations were originated by Aczél [1], Belousov [2], Neumann [3], and Taylor [4]. The main tool used to study hyperidentities and hypervarieties is the concept of a hypersubstitution which was introduced by Denecke et al. [5]. In 2000, Leeratanavalee and Denecke generalized the concept of hypersubstitution [6].

Let $X=\left\{x_{1}, x_{2}, x_{3}, \ldots\right\}$ be a countably infinite set of symbols called variables. We often refer to these variables as letters, to $X$ as an alphabet, and also refer to the set $X_{n}=\left\{x_{1}, x_{2}, \ldots, x_{n}\right\}$ as an $n$-element alphabet. Let $\left(f_{i}\right)_{i \in I}$ be an indexed set which is disjoint from $X$. Each $f_{i}$ is called $n_{i}$ ary operation symbol, where $n_{i} \geq 1$ is a natural number. Let $\tau$ be a function which assigns to every $f_{i}$ the number $n_{i}$ as its arity. The function $\tau$, on the values of $\tau$ written as $\left(n_{i}\right)_{i \in I}$ is called a type.
An $n$-ary term of type, $\tau$, or simply an $n$-ary term is defined inductively as follows:

(i) the variables $x_{1}, \ldots, x_{n}$ are $n$-ary terms.

(ii) If $t_{1}, \ldots, t_{n_{i}}$ are $n$-ary terms, then $f_{i}\left(t_{1}, \ldots, t_{n_{i}}\right)$ is an $n$-ary term.

By $W_{\tau}\left(X_{n}\right)$ we mean the smallest set which contains $x_{1}, \ldots, x_{n}$ and is closed under finite application of (ii). It is clear that every $n$-ary term is also an $m$-ary term for all $m \geq n$. The set $W_{\tau}(X):=\bigcup_{n=1}^{\infty} W_{\tau}\left(X_{n}\right)$ is the set of all terms of type $\tau$ over the alphabet $X$. This set can be used as the universe of an algebra of type $\tau$. For every $i \in I$, an $n_{i}$-ary operation $\overline{f_{i}}$ on $W_{\tau}(X)$ is defined by $\overline{f_{i}}: W_{\tau}(X)^{n_{i}} \rightarrow W_{\tau}(X)$ with $\left(t_{1}, \ldots, t_{n_{i}}\right) \mapsto f_{i}\left(t_{1}, \ldots, t_{n_{i}}\right)$. The algebra $\mathscr{F}_{\tau}(X):=$ $\left(W_{\tau}(X) ;\left(\overline{f_{i}}\right)_{i \in I}\right)$ is called the absolutely free algebra of type $\tau$ over the set $X$. The term algebra $\mathscr{F}_{\tau}(X)$ is generated by the set $X$ and has the property called absolute freeness, meaning that for every algebra $\mathscr{A} \in \operatorname{Alg}(\tau)$ and every mapping $f: X \rightarrow \mathscr{A}$ there exists a unique homomorphism $\widehat{f}: \mathscr{F}_{\tau}(X) \rightarrow \mathscr{A}$ which extends the mapping $f$ and such that $\widehat{f} \circ \varphi=f$, where $\varphi: X \rightarrow \mathscr{F}_{\tau}(X)$ is the embedding of $X$ into $\mathscr{F}_{\tau}(X)$. This can be shown by Figure 1 .

It is clear that the absolutely free algebras $\mathscr{F}_{\tau}(X)$ and $\mathscr{F}_{\tau}(Y)$ are isomorphic if $X$ and $Y$ have the same cardinality. One can use trees instead of terms. Trees are connected 


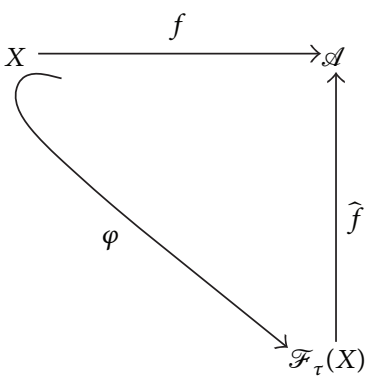

Figure 1: The embedding of $X$ into $\mathscr{F}_{\tau}(X)$.

graphs which have no cycles. It can be used to visualize the structure of computer programs. For applications, we can measure the complexity of a tree. Then the depth of a term (or the height of a tree) is defined. Let $t \in W_{\tau}(X)$, the depth of a term $t$ which is denoted by $\operatorname{depth}(t)$ and is defined as follows:

(i) if $t=x \in X$, then $\operatorname{depth}(t):=0$,

(ii) if $t=f_{i}\left(t_{1}, \ldots, t_{n_{i}}\right)$ where $t_{1}, \ldots, t_{n_{i}} \in W_{\tau}(X)$, then $\operatorname{depth}(t):=\max \left\{\operatorname{depth}\left(t_{j}\right) \mid 1 \leq j \leq n_{i}\right\}+1$.

A generalized hypersubstitution of type $\tau$ is a mapping $\sigma:\left\{f_{i} \mid i \in I\right\} \rightarrow W_{\tau}(X)$ which does not necessarily preserve the arity.

We denoted the set of all generalized hypersubstitutions of type $\tau$ by $\operatorname{Hyp}_{G}(\tau)$. To define a binary operation on $\operatorname{Hyp}_{G}(\tau)$, we need to define the concept of generalized superposition of terms $S^{m}: W_{\tau}(X)^{m+1} \rightarrow W_{\tau}(X)$ by the following steps.

For any term $t \in W_{\tau}(X)$,

(i) if $t=x_{j}, 1 \leq j \leq m$, then $S^{m}\left(x_{j}, t_{1}, \ldots, t_{m}\right):=t_{j}$,

(ii) if $t=x_{j}, m<j \in \mathbb{N}$, then $S^{m}\left(x_{j}, t_{1}, \ldots, t_{m}\right):=x_{j}$,

(iii) if $t=f_{i}\left(s_{1}, \ldots, s_{n_{i}}\right)$, then

$$
\begin{aligned}
& S^{m}\left(t, t_{1}, \ldots, t_{m}\right) \\
& \quad:=f_{i}\left(S^{m}\left(s_{1}, t_{1}, \ldots, t_{m}\right), \ldots, S^{m}\left(s_{n_{i}}, t_{1}, \ldots, t_{m}\right)\right) .
\end{aligned}
$$

Then the generalized hypersubstitution $\sigma$ can be extended to a mapping $\widehat{\sigma}: W_{\tau}(X) \rightarrow W_{\tau}(X)$ by the following steps:

(i) $\widehat{\sigma}[x]:=x \in X$,

(ii) $\widehat{\sigma}\left[f_{i}\left(t_{1}, \ldots, t_{n_{i}}\right)\right]:=S^{n_{i}}\left(\sigma\left(f_{i}\right), \widehat{\sigma}\left[t_{1}\right], \ldots, \widehat{\sigma}\left[t_{n_{i}}\right]\right)$, for any $n_{i}$-ary operation symbol $f_{i}$ where $\widehat{\sigma}\left[t_{j}\right], \quad 1 \leq j \leq$ $n_{i}$ are already defined.

We defined a binary operation ${ }^{\circ}$ on $\operatorname{Hyp}_{G}(\tau)$ by $\sigma_{1}{ }_{G} \sigma_{2}:=$ $\widehat{\sigma}_{1} \circ \sigma_{2}$, where $\circ$ denotes the usual composition of mappings and $\sigma_{1}, \sigma_{2} \in \operatorname{Hyp}_{G}(\tau)$. Let $\sigma_{\text {id }}$ be the hypersubstitution which maps each $n_{i}$-ary operation symbol $f_{i}$ to the term $f_{i}\left(x_{1}, \ldots, x_{n_{i}}\right)$. In [6], it is proved that for arbitrary terms $t, t_{1}, \ldots, t_{n} \in W_{\tau}(X)$ and for arbitrary generalized hypersubstitutions $\sigma, \sigma_{1}, \sigma_{2}$ we have (i) $S^{n}\left(\widehat{\sigma}[t], \widehat{\sigma}\left[t_{1}\right], \ldots, \widehat{\sigma}\left[t_{n}\right]\right)=\widehat{\sigma}\left[S^{n}\left(t, t_{1}, \ldots, t_{n}\right)\right]$,

(ii) $\left(\widehat{\sigma}_{1} \circ \sigma_{2}\right)=\widehat{\sigma}_{1} \circ \widehat{\sigma}_{2}$.

It turns out that $\left(\operatorname{Hyp}_{G}(\tau) ;{ }_{G}^{\circ}, \sigma_{\text {id }}\right)$ is a monoid where $\sigma_{\text {id }}$ is its identity element. The monoid $\left(\operatorname{Hyp}(\tau) ;{ }_{h}, \sigma_{\text {id }}\right)$ of all arity preserving hypersubstitutions of type $\tau$ forms a submonoid of $\left(\operatorname{Hyp}_{G}(\tau) ;{ }_{G}, \sigma_{\text {id }}\right)$. In 2001, Denecke et al. introduced the concept of the depth of a hypersubstitution [7]. Also in 2002, Denecke and Changpas defined full terms and studied full hypersubstitutions [8]. In this paper we generalized these concepts to the depth of generalized full terms and generalized full hypersubstitutions.

\section{Generalized Full Terms}

We use standard terminology established in modern algebra; see [9-11]. In this section, we give the definition of a generalized full term of type $\tau$, or simply a generalized full term, and describe the behavior of the depth of a generalized full term.

Definition 1. Let $\Omega$ be the set of operation symbols and $f \in \Omega$ with arity $(f)=n$ :

(i) if $s:\{1, \ldots, n\} \rightarrow\{1, \ldots, n\}$ is a permutation, then $f\left(x_{s(1)}, \ldots, x_{s(n)}\right)$ is a generalized full term,

(ii) if $j_{1}, j_{2}, \ldots, j_{n}$ are natural numbers greater than $n$, then $f\left(x_{s^{\prime}\left(j_{1}\right)}, \ldots, x_{s^{\prime}\left(j_{n}\right)}\right)$ is a generalized full term where $s^{\prime}$ is a permutation on $\left\{j_{1}, \ldots, j_{n}\right\}$,

(iii) if $t_{1}, \ldots, t_{n}$ are generalized full terms, then $f\left(t_{1}, \ldots, t_{n}\right)$ is a generalized full term.

Let $W_{(n)}^{\mathrm{GF}}(X)$ be the set of all generalized full terms and let $P_{n}$ be the set of all permutations on $\{1,2, \ldots, n\}$. By Definition 1 , the set $W_{(n)}^{\mathrm{GF}}(X)$ is closed under finite application of (iii). Therefore $\left(W_{(n)}^{\mathrm{GF}}(X) ;\left(\bar{f}_{i}\right)_{i \in I}\right)$ is a subalgebra of $\mathscr{F}_{\tau}(X)$.

Proposition 2. Let $p, p_{1}, p_{2}, \ldots, p_{n} \in W_{(n)}^{G F}(X)$. Then $S^{n}(p$, $\left.p_{1}, \ldots p_{n}\right)$ is also a generalized full term.

Proof. We give a proof by induction on the complexity of a generalized full term $p$.

If $p=f\left(x_{s(1)}, \ldots, x_{s(n)}\right)$ where $s \in P_{n}$, then:

$$
\begin{aligned}
S^{n}\left(p, p_{1}, \ldots, p_{n}\right) & =S^{n}\left(f_{i}\left(x_{s(1)}, \ldots, x_{s(n)}\right), p_{1}, \ldots, p_{n}\right) \\
& =f\left(p_{s(1)}, \ldots, p_{s(n)}\right)
\end{aligned}
$$

which is a generalized full term.

If $p=f\left(x_{s^{\prime}\left(j_{1}\right)}, \ldots, x_{s^{\prime}\left(j_{n}\right)}\right)$ where $j_{1}, \ldots j_{n}$ are natural numbers greater than $n$ and $s^{\prime}$ is a permutation on $\left\{j_{1}, \ldots, j_{n}\right\}$, then

$$
\begin{aligned}
S^{n}\left(p, p_{1}, \ldots, p_{n}\right) & =S^{n}\left(f\left(x_{s^{\prime}\left(j_{1}\right)}, \ldots, x_{s^{\prime}\left(j_{n}\right)}\right), p_{1}, \ldots, p_{n}\right) \\
& =f\left(x_{s^{\prime}\left(j_{1}\right)}, \ldots, x_{s^{\prime}\left(j_{n}\right)}\right)
\end{aligned}
$$

which is a generalized full term. 
If $p=f\left(t_{1}, \ldots, t_{n}\right)$ where $t_{1}, \ldots, t_{n} \in W_{(n)}^{\mathrm{GF}}(X)$ and assume that $S^{n}\left(t_{j}, p_{1}, \ldots, p_{n}\right)$ are generalized full terms for all $1 \leq j \leq n$, then

$$
\begin{aligned}
S^{n}(p & \left.p_{1}, \ldots, p_{n}\right) \\
& =S^{n}\left(f\left(t_{1}, \ldots, t_{n}\right), p_{1}, \ldots, p_{n}\right) \\
& =f\left(S^{n}\left(t_{1}, p_{1}, \ldots, p_{n}\right), \ldots, S^{n}\left(t_{n}, p_{1}, \ldots, p_{n}\right)\right) .
\end{aligned}
$$

By Definition 1(iii), $S^{n}\left(p, p_{1}, \ldots, p_{n}\right)$ is a generalized full term.

Now we consider the algebra clone ${ }_{\mathrm{GF}}(n):=\left(W_{(n)}^{\mathrm{GF}}(X) ; S^{n}\right)$ of type $(n+1)$ with $\mathscr{F}_{\mathrm{GS}(n)}=\left\{f\left(x_{s(1)}, \ldots, x_{s(n)}\right) \mid s \in P_{n}\right\} \cup$ $\left\{f\left(x_{s^{\prime}\left(j_{1}\right)}, \ldots, x_{s^{\prime}\left(j_{n}\right)}\right) \mid j_{1}, \ldots, j_{n}>n, s^{\prime}\right.$ is a permutation on $\left.\left\{j_{1}, \ldots, j_{n}\right\}\right\}$ as a generating system. The algebra clone ${ }_{\mathrm{GF}}(n)$ is called the clone of generalized full terms of type $(n)$. Then we have the following.

Proposition 3. The algebra clone ${ }_{G F}(n)$ satisfies the so-called superassociative law $(C)$

$$
\begin{gathered}
\widetilde{S}^{n}\left(X_{0}, \widetilde{S}^{n}\left(Y_{1}, X_{1}, \ldots, X_{n}\right), \ldots, \widetilde{S}^{n}\left(Y_{n}, X_{1}, \ldots, X_{n}\right)\right) \\
\approx \widetilde{S}^{n}\left(\widetilde{S}^{n}\left(X_{0}, Y_{1}, \ldots, Y_{n}\right), X_{1}, \ldots, X_{n}\right),
\end{gathered}
$$

where $\widetilde{S}^{n}$ is an $(n+1)$-ary operation symbol and $X_{l}, Y_{j}$ are variables.

Proof. We give a proof by induction on the complexity of the generalized full term which is substituted for $X_{0}$. Substituting for $X_{0}$ a term of the form $f\left(x_{s(1)}, \ldots, x_{s(n)}\right)$ for $s \in P_{n}$, then

$$
\begin{aligned}
S^{n}( & f\left(x_{s(1)}, \ldots, x_{s(n)}\right), S^{n}\left(t_{1}, s_{1}, \ldots, s_{n}\right), \ldots, \\
& \left.S^{n}\left(t_{n}, s_{1}, \ldots, s_{n}\right)\right) \\
= & f\left(S^{n}\left(x_{s(1)}, S^{n}\left(t_{1}, s_{1}, \ldots, s_{n}\right), \ldots, S^{n}\left(t_{n}, s_{1}, \ldots, s_{n}\right)\right), \ldots,\right. \\
& \left.S^{n}\left(x_{s(n)}, S^{n}\left(t_{1}, s_{1}, \ldots, s_{n}\right), \ldots, S^{n}\left(t_{n}, s_{1}, \ldots, s_{n}\right)\right)\right) \\
= & f\left(S^{n}\left(t_{s(1)}, s_{1}, \ldots, s_{n}\right), \ldots, S^{n}\left(t_{s(n)}, s_{1}, \ldots, s_{n}\right)\right) \\
= & S^{n}\left(f\left(t_{s(1)}, \ldots, t_{s(n)}\right), s_{1}, \ldots, s_{n}\right) \\
= & S^{n}\left(S^{n}\left(f\left(x_{s(1)}, \ldots, x_{s(n)}\right), t_{1}, \ldots, t_{n}\right), s_{1}, \ldots, s_{n}\right) .
\end{aligned}
$$

If we substitute for $X_{0}$ a term $t=f\left(x_{s^{\prime}\left(j_{1}\right)}, \ldots, x_{s^{\prime}\left(j_{n}\right)}\right)$ where $j_{1}, \ldots, j_{n}$ are natural numbers greater than $n$ and $s^{\prime}$ is a permutation on $\left\{j_{1}, \ldots, j_{n}\right\}$, then

$$
\begin{aligned}
S^{n}( & f\left(x_{s^{\prime}\left(j_{1}\right)}, \ldots, x_{s^{\prime}\left(j_{n}\right)}\right), S^{n}\left(t_{1}, s_{1}, \ldots, s_{n}\right), \ldots, \\
& \left.S^{n}\left(t_{n}, s_{1}, \ldots, s_{n}\right)\right) \\
= & f\left(x_{s^{\prime}\left(j_{1}\right)}, \ldots, x_{s^{\prime}\left(j_{n}\right)}\right) \\
= & S^{n}\left(f\left(x_{s^{\prime}\left(j_{1}\right)}, \ldots, x_{s^{\prime}\left(j_{n}\right)}\right), s_{1}, \ldots, s_{n}\right) \\
= & S^{n}\left(f \left(S^{n}\left(x_{s^{\prime}\left(j_{1}\right)}, t_{1}, \ldots, t_{n}\right), \ldots,\right.\right.
\end{aligned}
$$

$$
\begin{gathered}
\left.\left.S^{n}\left(x_{s^{\prime}\left(j_{n}\right)}, t_{1}, \ldots, t_{n}\right)\right), s_{1}, \ldots, s_{n}\right) \\
=S^{n}\left(S^{n}\left(f\left(x_{s^{\prime}\left(j_{1}\right)}, \ldots, x_{s^{\prime}\left(j_{n}\right)}\right), t_{1}, \ldots, t_{n}\right), s_{1}, \ldots, s_{n}\right) .
\end{gathered}
$$

If we substitute for $X_{0}$ a term $t=f\left(r_{1}, \ldots, r_{n}\right)$ and assume that $(C)$ is satisfied for $r_{1}, \ldots, r_{n} \in W_{(n)}^{\mathrm{GF}}(X)$, then

$$
\begin{aligned}
S^{n}( & \left.f\left(r_{1}, \ldots, r_{n}\right), S^{n}\left(t_{1}, s_{1}, \ldots, s_{n}\right), \ldots, S^{n}\left(t_{n}, s_{1}, \ldots, s_{n}\right)\right) \\
= & f\left(S^{n}\left(r_{1}, S^{n}\left(t_{1}, s_{1}, \ldots, s_{n}\right), \ldots, S^{n}\left(t_{n}, s_{1}, \ldots, s_{n}\right)\right), \ldots,\right. \\
& \left.S^{n}\left(r_{n}, S^{n}\left(t_{1}, s_{1}, \ldots, s_{n}\right), \ldots, S^{n}\left(t_{n}, s_{1}, \ldots, s_{n}\right)\right)\right) \\
= & S^{n}\left(f\left(S^{n}\left(r_{1}, t_{1}, \ldots, t_{n}\right), \ldots, S^{n}\left(r_{n}, t_{1}, \ldots, t_{n}\right)\right),\right. \\
& \left.s_{1}, \ldots, s_{n}\right) \\
= & S^{n}\left(S^{n}\left(f\left(r_{1}, \ldots, r_{n}\right), t_{1}, \ldots, t_{n}\right), s_{1}, \ldots, s_{n}\right) .
\end{aligned}
$$

An algebra $\left(M ; S^{n}\right)$ of type $(n+1)$ which satisfies the condition (C) is called a Menger algebra of rank $n$.

Lemma 4. Let $t, t_{1}, t_{2}, \ldots, t_{n} \in W_{(n)}^{G F}(X)$. If $\operatorname{depth}(t)=1$, then

$$
\begin{gathered}
\operatorname{depth}\left(S^{n}\left(t, t_{1}, \ldots, t_{n}\right)\right)=1 \\
\text { or } \max \left\{\operatorname{depth}\left(t_{1}\right), \ldots, \operatorname{depth}\left(t_{n}\right)\right\}+1 \text {. }
\end{gathered}
$$

Proof. Assume that depth $(t)=1$. Then $t=f\left(x_{s(1)}, \ldots, x_{s(n)}\right)$ where $s \in P_{n}$ or $t=f\left(x_{s^{\prime}\left(j_{1}\right)}, \ldots, x_{s^{\prime}\left(j_{n}\right)}\right)$ where $j_{1}, \ldots, j_{n}$ are natural numbers greater than $n$ and $s^{\prime}$ is a permutation on $\left\{j_{1}, \ldots, j_{n}\right\}$ :

if $t=f\left(x_{s(1)}, \ldots, x_{s(n)}\right)$, then

$$
\begin{aligned}
\operatorname{depth} & \left(S^{n}\left(t, t_{1}, \ldots, t_{n}\right)\right) \\
& =\operatorname{depth}\left(S^{n}\left(f\left(x_{s(1)}, \ldots, x_{s(n)}\right), t_{1}, \ldots, t_{n}\right)\right) \\
& =\operatorname{depth}\left(f\left(t_{s(1)}, \ldots, t_{s(n)}\right)\right) \\
& =\max \left\{\operatorname{depth}\left(t_{s(1)}\right), \ldots, \operatorname{depth}\left(t_{s(n)}\right)\right\}+1 \\
& =\max \left\{\operatorname{depth}\left(t_{1}\right), \ldots, \operatorname{depth}\left(t_{n}\right)\right\}+1 .
\end{aligned}
$$

If $t=f\left(x_{s^{\prime}\left(j_{1}\right)}, \ldots, x_{s^{\prime}\left(j_{n}\right)}\right)$, then

$$
\begin{aligned}
& \operatorname{depth}\left(S^{n}\left(t, t_{1}, \ldots, t_{n}\right)\right) \\
& =\operatorname{depth}\left(S^{n}\left(f\left(x_{s^{\prime}\left(j_{1}\right)}, \ldots, x_{s\left(j_{n}\right)}^{\prime}\right), t_{1}, \ldots, t_{n}\right)\right) \\
& =\operatorname{depth}\left(f\left(x_{s^{\prime}\left(j_{1}\right)}, \ldots, x_{s^{\prime}\left(j_{n}\right)}\right)\right) \\
& =1 .
\end{aligned}
$$

Theorem 5. Let $t, t_{1}, t_{2}, \ldots, t_{n} \in W_{(n)}^{G F}(X)$. Then $\operatorname{depth}\left(S^{n}(t\right.$, $\left.\left.t_{1}, \ldots, t_{n}\right)\right)=1$ or $\max \left\{\operatorname{depth}\left(t_{1}\right), \ldots, \operatorname{depth}\left(t_{n}\right)\right\}+\operatorname{depth}(t)$. 
Proof. We prove this fact by induction on the complexity of $\operatorname{depth}(t)$.

If $\operatorname{depth}(t)=1$, then by Lemma 4 we have $\operatorname{depth}\left(S^{n}(t\right.$, $\left.\left.t_{1}, \ldots, t_{n}\right)\right)=1$ or $\max \left\{\operatorname{depth}\left(t_{1}\right), \ldots, \operatorname{depth}\left(t_{n}\right)\right\}+\operatorname{depth}(t)$.

If $\operatorname{depth}(t)>1$, then we have $t=f\left(s_{1}, \ldots, s_{n}\right)$ and assume that the formula is satisfied for $s_{1}, \ldots, s_{n} \in W_{(n)}^{\mathrm{GF}}(X)$ that is $\operatorname{depth}\left(S^{n}\left(s_{k}, t_{1}, \ldots, t_{n}\right)\right)=1$ or $\max \left\{\operatorname{depth}\left(t_{1}\right), \ldots\right.$, $\left.\operatorname{depth}\left(t_{n}\right)\right\}+\operatorname{depth}\left(s_{k}\right)$ for all $1 \leq k \leq n$. Then we have

$$
\begin{aligned}
& \operatorname{depth}\left(S^{n}\left(t, t_{1}, \ldots, t_{n}\right)\right) \\
&=\operatorname{depth}\left(f\left(S^{n}\left(s_{1}, t_{1}, \ldots, t_{n}\right), \ldots, S^{n}\left(s_{n}, t_{1}, \ldots, t_{n}\right)\right)\right) \\
&=\max \left\{\operatorname{depth}\left(S^{n}\left(s_{1}, t_{1}, \ldots, t_{n}\right)\right), \ldots,\right. \\
&\left.\quad \operatorname{depth}\left(S^{n}\left(s_{n}, t_{1}, \ldots, t_{n}\right)\right)\right\}+1 \\
&=\max \left\{\max \left\{\operatorname{depth}\left(t_{1}\right), \ldots, \operatorname{depth}\left(t_{n}\right)\right\}\right. \\
& \quad+\operatorname{depth}\left(s_{1}\right), \ldots, \\
& \quad \max \left\{\operatorname{depth}\left(t_{1}\right), \ldots, \operatorname{depth}\left(t_{n}\right)\right\} \\
&\left.\quad+\operatorname{depth}\left(s_{n}\right)\right\}+1 \\
&=\max \left\{\operatorname{depth}\left(t_{1}\right), \ldots, \operatorname{depth}\left(t_{n}\right)\right\} \\
& \quad+\max \left\{\operatorname{depth}\left(s_{1}\right), \ldots, \operatorname{depth}\left(s_{n}\right)\right\}+1 \\
&=\max \left\{\operatorname{depth}\left(t_{1}\right), \ldots, \operatorname{depth}\left(t_{n}\right)\right\}+\operatorname{depth}(t) .
\end{aligned}
$$

\section{The Depth of Generalized Full Terms with respect to $k$}

In this section, we generalize the concept of the depth of a full term $t$ with respect to $k$ which were studied by Denecke et al. [7] to the depth of a generalized full term $t$ with respect to $k$.

Definition 6. Let $t \in W_{(n)}^{\mathrm{GF}}(X)$ and let $k$ be a fixed element of $\{1,2, \ldots, n\}$ and $\operatorname{var}(t)$ be the set of all variables occurring in $t$. For each variable $x_{k}$, the depth of a generalized full term $t$ with respect to $k$ denoted by $\operatorname{depth}_{k}(t)$ is defined inductively as follows:

(i) if $t=f\left(x_{s(1)}, \ldots, x_{s(n)}\right)$ where $s \in P_{n}$, then $\operatorname{depth}_{k}(t)=$ 1 ,

(ii) if $t=f\left(x_{s^{\prime}\left(j_{1}\right)}, \ldots, x_{s^{\prime}\left(j_{n}\right)}\right)$ where $j_{1}, j_{2}, \ldots, j_{n}$ are natural numbers greater than $n$ and $s^{\prime}$ is a permutation on $\left\{j_{1}, \ldots, j_{n}\right\}$, then $\operatorname{depth}_{k}(t)=0$,

(iii) if $t=f\left(t_{1}, \ldots, t_{n}\right)$ and assume that $\operatorname{depth}_{k}\left(t_{j}\right), 1 \leq j \leq$ $n$ are already defined, then

$$
\begin{aligned}
& \operatorname{depth}_{k}(t) \\
& =\left\{\begin{aligned}
0, & \text { if } x_{k} \notin \operatorname{var}(t) ; \\
\max \left\{\operatorname{depth}_{k}\left(t_{j}\right) \mid 1 \leq j \leq n ;\right. & \text { otherwise } \\
\left.x_{k} \in \operatorname{var}\left(t_{j}\right)\right\}+1 . &
\end{aligned}\right.
\end{aligned}
$$

Theorem 7. Let $t, t_{1}, t_{2}, \ldots, t_{n} \in W_{(n)}^{G F}(X)$. Then

$$
\begin{aligned}
& \operatorname{depth}\left(S^{n}\left(t, t_{1}, \ldots, t_{n}\right)\right) \\
& \quad \geq \max \left\{\operatorname{depth}_{j}(t)+\operatorname{depth}\left(t_{j}\right) \mid 1 \leq j \leq n ; x_{j} \in \operatorname{var}(t)\right\} .
\end{aligned}
$$

Proof. We prove this fact by induction on the complexity of $\operatorname{depth}(t)$. Suppose that $\operatorname{depth}(t)=1$. Then $t=f\left(x_{s(1)}\right.$, $\left.\ldots, x_{s(n)}\right)$ where $s \in P_{n}$ or $t=f\left(x_{s^{\prime}\left(j_{1}\right)}, \ldots, x_{s^{\prime}\left(j_{n}\right)}\right)$ where $j_{1}, j_{2}, \ldots, j_{n}$ are natural numbers greater than $n$, and $s^{\prime}$ is a permutation on $\left\{j_{1}, \ldots, j_{n}\right\}$.

If $t=f\left(x_{s(1)}, \ldots, x_{s(n)}\right)$, then $S^{n}\left(t, t_{1}, \ldots, t_{n}\right)=S^{n}\left(f\left(x_{s(1)}\right.\right.$, $\left.\left.\ldots, x_{s(n)}\right), t_{1}, \ldots, t_{n}\right)=f\left(t_{s(1)}, \ldots, t_{s(n)}\right)$. Therefore,

$$
\begin{aligned}
\operatorname{depth} & \left(S^{n}\left(t, t_{1}, \ldots, t_{n}\right)\right) \\
= & \operatorname{depth}\left(f\left(t_{s(1)}, \ldots, t_{s(n)}\right)\right) \\
= & \max \left\{\operatorname{depth}\left(t_{s(1)}\right), \ldots, \operatorname{depth}\left(t_{s(n)}\right)\right\}+1, \\
\max & \left\{\operatorname{depth}_{j}(t)+\operatorname{depth}\left(t_{j}\right) \mid 1 \leq j \leq n ; x_{j} \in \operatorname{var}(t)\right\} \\
= & \max \left\{1+\operatorname{depth}\left(t_{1}\right), \ldots, 1+\operatorname{depth}\left(t_{n}\right)\right\} \\
= & \max \left\{\operatorname{depth}\left(t_{s(1)}\right), \ldots, \operatorname{depth}\left(t_{s(n)}\right)\right\}+1 .
\end{aligned}
$$

If $t=f\left(x_{s^{\prime}\left(j_{1}\right)}, \ldots, x_{s^{\prime}\left(j_{n}\right)}\right)$, then $S^{n}\left(t, t_{1}, \ldots, t_{n}\right)=S^{n}\left(f\left(x_{s^{\prime}\left(j_{1}\right)}\right.\right.$, $\left.\left.\ldots, x_{s^{\prime}\left(j_{n}\right)}\right), t_{1}, \ldots, t_{n}\right)=f\left(x_{s^{\prime}\left(j_{1}\right)}, \ldots, x_{s^{\prime}\left(j_{n}\right)}\right)$. Therefore,

$$
\begin{aligned}
\operatorname{depth}\left(S^{n}\left(t, t_{1}, \ldots, t_{n}\right)\right) & =\operatorname{depth}\left(f\left(x_{s^{\prime}\left(j_{1}\right)}, \ldots, x_{s^{\prime}\left(j_{n}\right)}\right)\right) \\
& =1,
\end{aligned}
$$

$\max \left\{\operatorname{depth}_{j}(t)+\operatorname{depth}\left(t_{j}\right) \mid 1 \leq j \leq n ; x_{j} \in \operatorname{var}(t)\right\}=0$.

If $\operatorname{depth}(t)>1$, then $t=f\left(p_{1}, \ldots, p_{n}\right)$ where $p_{1}, \ldots, p_{n} \in$ $W_{(n)}^{\mathrm{GF}}(X)$ and assume that the formula is satisfied for $p_{1}, \ldots$, $p_{n}$. Thus

$$
\begin{aligned}
S^{n}(t, & \left.t_{1}, \ldots, t_{n}\right) \\
& =S^{n}\left(f\left(p_{1}, \ldots, p_{n}\right), t_{1}, \ldots, t_{n}\right) \\
& =f\left(S^{n}\left(p_{1}, t_{1}, \ldots, t_{n}\right), \ldots, S^{n}\left(p_{n}, t_{1}, \ldots, t_{n}\right)\right),
\end{aligned}
$$

$\operatorname{depth}\left(S^{n}\left(t, t_{1}, \ldots, t_{n}\right)\right)$

$=\max \left\{\operatorname{depth}\left(S^{n}\left(p_{1}, t_{1}, \ldots, t_{n}\right)\right), \ldots\right.$,

$\left.\operatorname{depth}\left(S^{n}\left(p_{n}, t_{1}, \ldots, t_{n}\right)\right)\right\}+1$ 


$$
\begin{aligned}
& \geq \max \left\{\operatorname { m a x } \left\{\operatorname{depth}_{j}\left(p_{1}\right)\right.\right. \\
& \left.+\operatorname{depth}\left(t_{j}\right) \mid 1 \leq j \leq n ; x_{j} \in \operatorname{var}\left(p_{1}\right)\right\}, \ldots, \\
& \max \left\{\operatorname{depth}_{j}\left(p_{n}\right)\right. \\
& \left.\left.\left.+\operatorname{depth}_{j}\right) \mid 1 \leq j \leq n ; x_{j} \in \operatorname{var}\left(p_{n}\right)\right\}\right\}+1 \\
& =\max \left\{\operatorname { m a x } \left\{\operatorname{depth}_{j}\left(p_{k}\right) \mid 1 \leq j \leq n,\right.\right. \\
& \left.1 \leq k \leq n ; x_{j} \in \operatorname{var}\left(p_{k}\right)\right\}+1 \\
& +\operatorname{depth}\left(t_{j}\right) \mid 1 \leq j \leq n, \\
& \left.x_{j} \in \cup\left\{\operatorname{var}\left(p_{k}\right) \mid 1 \leq k \leq n\right\}\right\} \\
& =\max \left\{\operatorname { m a x } _ { 2 } \left\{\operatorname{depth}_{j}\left(p_{k}\right) \mid 1 \leq j \leq n,\right.\right. \\
& \left.1 \leq k \leq n ; x_{j} \in \operatorname{var}\left(p_{k}\right)\right\}+1 \\
& \left.\left.+\operatorname{depth}\left(t_{j}\right) \mid 1 \leq j \leq n ; x_{j} \in \operatorname{var}(t)\right\}\right\} \\
& =\max \left\{\operatorname{depth}_{j}(t)+\operatorname{depth}\left(t_{j}\right) \mid 1 \leq j \leq n ; x_{j} \in \operatorname{var}(t)\right\} .
\end{aligned}
$$

Example 8. Let $\tau=$ (3) be a type; that is, we have only one ternary operation symbol, say $f$. Let $t, t_{1}, t_{2} \in W_{(3)}^{\mathrm{GF}}(X)$ where $t=f\left(f\left(x_{2}, x_{1}, x_{3}\right), f\left(x_{7}, x_{4}, x_{5}\right), f\left(x_{3}, x_{2}, x_{1}\right)\right), t_{1}=f\left(x_{9}\right.$, $\left.x_{5}, x_{4}\right), t_{2}=f\left(f\left(x_{2}, x_{3}, x_{1}\right), f\left(x_{2}, x_{3}, x_{1}\right), f\left(x_{2}, x_{3}, x_{1}\right)\right)$ and $t_{3}=f\left(x_{6}, x_{4}, x_{5}\right)$. Then we have $\operatorname{depth}(t)=2, \operatorname{depth}\left(t_{1}\right)=$ 1 , $\operatorname{depth}\left(t_{2}\right)=2$, and $\operatorname{depth}\left(t_{3}\right)=1$. Thus $\operatorname{depth}\left(S^{3}\left(t, t_{1}\right.\right.$, $\left.\left.t_{2}, t_{3}\right)\right)=4$. Consider $\max \left\{\operatorname{depth}_{1}(t)+\operatorname{depth}\left(t_{1}\right), \operatorname{depth}_{2}(t)+\right.$ $\operatorname{depth}\left(t_{2}\right)$, and $\left.\operatorname{depth}_{3}(t)+\operatorname{depth}\left(t_{3}\right)\right\}=\max \{2+1,2+2,2+$ $1\}=4$.

\section{Generalized Full Hypersubstitutions and Substitutions of clone $_{\mathrm{GF}}(n)$}

In this section, we will generalize the concept of full hypersubstitution. For any generalized full term $t$, we define the generalized full term $t_{s}$ arising from $t$ by mapping all variables corresponding to a permutation $s \in P_{n}$ inductively by the following steps:

(i) if $t=f\left(x_{r(1)}, \ldots, x_{r(n)}\right)$ where $r \in P_{n}$, then $t_{s}=$ $f\left(x_{s(r(1))}, \ldots, x_{s(r(n))}\right)$,

(ii) if $t=f\left(x_{s^{\prime}\left(j_{1}\right)}, \ldots, x_{s^{\prime}\left(j_{n}\right)}\right)$ where $j_{1}, \ldots, j_{n}$ are natural numbers greater than $n$ and $s^{\prime}$ is a permutation on $\left\{j_{1}, \ldots, j_{n}\right\}$, then $t_{s}=f\left(x_{s^{\prime}\left(j_{1}\right)}, \ldots, x_{s^{\prime}\left(j_{n}\right)}\right)$,

(iii) if $t=f\left(t_{1}, \ldots, t_{n}\right)$ where $t_{1}, \ldots, t_{n} \in W_{(n)}^{\mathrm{GF}}(X)$, then $t_{s}=f\left(\left(t_{1}\right)_{s}, \ldots,\left(t_{n}\right)_{s}\right)$.

It is clear that $t_{s}$ is a generalized full term for any generalized full term $t$ and for any $s \in P_{n}$.

Definition 9. A mapping $\sigma: \Omega \rightarrow W_{(n)}^{\mathrm{GF}}(X)$ is called a generalized full hypersubstitution.
By $\operatorname{Hyp}_{\mathrm{GF}}(n)$ we denote the set of all generalized full hypersubstitutions of type $(n)$. Every generalized full hypersubstitution $\sigma$ can be extended to a map $\widehat{\sigma}$ defined on $W_{(n)}^{\mathrm{GF}}(X)$ as follows:

(i) $\widehat{\sigma}\left[f\left(x_{s(1)}, \ldots, x_{s(n)}\right)\right]:=\sigma(f)_{s}$,

(ii) $\widehat{\sigma}\left[f\left(x_{s^{\prime}\left(j_{1}\right)}, \ldots, x_{s^{\prime}\left(j_{n}\right)}\right)\right]:=S^{n}\left(\sigma(f), x_{s^{\prime}\left(j_{1}\right)}, \ldots, x_{s^{\prime}\left(j_{n}\right)}\right)$,

(iii) $\widehat{\sigma}\left[f_{i}\left(t_{1}, \ldots, t_{n}\right)\right]:=S^{n}\left(\sigma(f), \widehat{\sigma}\left[t_{1}\right], \ldots, \widehat{\sigma}\left[t_{n}\right]\right)$.

We define a binary operation ${ }^{\circ} \mathrm{GF}$ on $\operatorname{Hyp}_{\mathrm{GF}}(n)$ by $\sigma_{1}{ }^{\circ}{ }_{\mathrm{GF}} \sigma_{2}:=$ $\widehat{\sigma}_{1} \circ \sigma_{2}$, where $\circ$ denotes the usual composition of functions. Together with the hypersubstitution $\sigma_{\text {id }}$ defined by $\sigma_{\text {id }}(f):=$ $f\left(x_{1}, \ldots, x_{n}\right)$, one has a monoid $\left(\operatorname{Hyp}_{\mathrm{GF}}(n) ;{ }_{\mathrm{GF}}, \sigma_{\mathrm{id}}\right)$. Then we have the following.

Lemma 10. Let $t \in W_{(n)}^{G F}(X), s \in P_{n}$, and $\sigma \in H y p_{G F}(n)$. Then

$$
S^{n}\left(t, \widehat{\sigma}\left[t_{s(1)}\right], \ldots, \widehat{\sigma}\left[t_{s(n)}\right]\right)=S^{n}\left(t_{s}, \widehat{\sigma}\left[t_{1}\right], \ldots, \widehat{\sigma}\left[t_{n}\right]\right)
$$

Proof. We give a proof by induction on the complexity of a generalized full term $t$.

$$
\text { If } t=f\left(x_{r(1)}, \ldots, x_{r(n)}\right) \text { where } r \in P_{n} \text {, then }
$$

$$
\begin{aligned}
S^{n}(t, & \left.\widehat{\sigma}\left[t_{s(1)}\right], \ldots, \widehat{\sigma}\left[t_{s(n)}\right]\right) \\
& =S^{n}\left(f\left(x_{r(1)}, \ldots, x_{r(n)}\right), \widehat{\sigma}\left[t_{s(1)}\right], \ldots, \widehat{\sigma}\left[t_{s(n)}\right]\right) \\
& =f\left(\widehat{\sigma}\left[t_{s(r(1))}\right], \ldots, \widehat{\sigma}\left[t_{s(r(n))}\right]\right), \\
S^{n}\left(t_{s}, \widehat{\sigma}\left[t_{1}\right], \ldots, \widehat{\sigma}\left[t_{n}\right]\right) & \\
& =S^{n}\left(f\left(x_{r(1)}, \ldots, x_{r(n)}\right)_{s}, \widehat{\sigma}\left[t_{1}\right], \ldots, \widehat{\sigma}\left[t_{n}\right]\right) \\
& =S^{n}\left(f\left(x_{s(r(1))}, \ldots, x_{s(r(n))}\right), \widehat{\sigma}\left[t_{1}\right], \ldots, \widehat{\sigma}\left[t_{n}\right]\right) \\
& =f\left(\widehat{\sigma}\left[t_{s(r(1))}\right], \ldots, \widehat{\sigma}\left[t_{s(r(n))}\right]\right) .
\end{aligned}
$$

If $t=f\left(x_{s^{\prime}\left(j_{1}\right)}, \ldots, x_{s^{\prime}\left(j_{n}\right)}\right)$ where $j_{1}, \ldots, j_{n}$ are natural numbers greater than $n$ and $s^{\prime}$ is a permutation on $\left\{j_{1}, \ldots, j_{n}\right\}$, then

$$
\begin{aligned}
S^{n}(t, & \left.\widehat{\sigma}\left[t_{s(1)}\right], \ldots, \widehat{\sigma}\left[t_{s(n)}\right]\right) \\
& =S^{n}\left(f\left(x_{s^{\prime}\left(j_{1}\right)}, \ldots, x_{s^{\prime}\left(j_{n}\right)}\right), \widehat{\sigma}\left[t_{s(1)}\right], \ldots, \widehat{\sigma}\left[t_{s(n)}\right]\right) \\
& =f\left(x_{s^{\prime}\left(j_{1}\right)}, \ldots, x_{s^{\prime}\left(j_{n}\right)}\right), \\
S^{n}\left(t_{s},\right. & \left.\widehat{\sigma}\left[t_{1}\right], \ldots, \widehat{\sigma}\left[t_{n}\right]\right) \\
& =S^{n}\left(\left(f\left(x_{s^{\prime}\left(j_{1}\right)}, \ldots, x_{s^{\prime}\left(j_{n}\right)}\right), \widehat{s}\left[t_{1}\right], \ldots, \widehat{\sigma}\left[t_{n}\right]\right)\right. \\
& =f\left(x_{s^{\prime}\left(j_{1}\right)}, \ldots, x_{s^{\prime}\left(j_{n}\right)}\right) .
\end{aligned}
$$


If $t=f\left(t_{1}^{\prime}, \ldots, t_{n}^{\prime}\right)$ where $t_{1}^{\prime}, \ldots, t_{n}^{\prime} \in W_{(n)}^{\mathrm{GF}}(X)$ and assume that the formula is satisfied for $t_{1}^{\prime}, \ldots, t_{n}^{\prime}$, then

$$
\begin{aligned}
S^{n}(t, \widehat{\sigma} & {\left.\left[t_{s(1)}\right], \ldots, \widehat{\sigma}\left[t_{s(n)}\right]\right) } \\
= & \left.S^{n}\left(f\left(t_{1}^{\prime}, \ldots, t_{n}^{\prime}\right)\right), \widehat{\sigma}\left[t_{s(1)}\right], \ldots, \widehat{\sigma}\left[t_{s(n)}\right]\right) \\
= & f\left(S^{n}\left(t_{1}^{\prime}, \widehat{\sigma}\left[t_{s(1)}\right], \ldots, \widehat{\sigma}\left[t_{s(n)}\right]\right), \ldots,\right. \\
& \left.S^{n}\left(t_{n}^{\prime}, \widehat{\sigma}\left[t_{s(1)}\right], \ldots, \widehat{\sigma}\left[t_{s(n)}\right]\right)\right) \\
= & f\left(S^{n}\left(\left(t_{1}^{\prime}\right)_{s}, \widehat{\sigma}\left[t_{1}\right], \ldots, \widehat{\sigma}\left[t_{n}\right]\right), \ldots,\right. \\
& \left.S^{n}\left(\left(t_{n}^{\prime}\right)_{s}, \widehat{\sigma}\left[t_{1}\right], \ldots, \widehat{\sigma}\left[t_{n}\right]\right)\right) \\
= & S^{n}\left(f\left(\left(t_{1}^{\prime}\right)_{s}, \ldots,\left(t_{n}^{\prime}\right)_{s}\right), \widehat{\sigma}\left[t_{1}\right], \ldots, \widehat{\sigma}\left[t_{n}\right]\right) \\
= & S^{n}\left(\left(f\left(t_{1}^{\prime}, \ldots, t_{n}^{\prime}\right)\right)_{s}, \widehat{\sigma}\left[t_{1}\right], \ldots, \widehat{\sigma}\left[t_{n}\right]\right) \\
= & S^{n}\left(t_{s}, \widehat{\sigma}\left[t_{1}\right], \ldots, \widehat{\sigma}\left[t_{n}\right]\right) .
\end{aligned}
$$

Lemma 11. Let $\sigma \in H y p_{G F}(n)$ and $p$ be a generalized full term. Then $\widehat{\sigma}[p]$ is also a generalized full term.

Proof. We give a proof by induction on the complexity of a generalized full term $p$.

If $p=f\left(x_{s(1)}, \ldots, x_{s(n)}\right)$ where $s \in P_{n}$, then $\widehat{\sigma}[p]=$ $\sigma(f)_{s}$. Since $\sigma(f)_{s}$ is a generalized full term, $\widehat{\sigma}[p]$ is also a generalized full term.

If $p=f\left(x_{s^{\prime}\left(j_{1}\right)}, \ldots, x_{s^{\prime}\left(j_{n}\right)}\right)$ where $j_{1}, \ldots, j_{n}$ are natural numbers greater than $n$ and $s^{\prime}$ is a permutation on $\left\{j_{1}, \ldots\right.$, $\left.j_{n}\right\}$, then $\widehat{\sigma}[p]=\widehat{\sigma}\left[f\left(x_{s^{\prime}\left(j_{1}\right)}, \ldots, x_{s^{\prime}\left(j_{n}\right)}\right)\right]=S^{n}\left(\sigma(f), x_{s^{\prime}\left(j_{1}\right)}\right.$, $\left.\ldots, x_{s^{\prime}\left(j_{n}\right)}\right)$. Since $S^{n}\left(\sigma(f), x_{s^{\prime}\left(j_{1}\right)}, \ldots, x_{s^{\prime}\left(j_{n}\right)}\right)$ is a generalized full term, $\widehat{\sigma}[p]$ is also a generalized full term.

If $p=f\left(p_{1}, \ldots, p_{n}\right)$ where $p_{j}$ are generalized full terms for all $1 \leq j \leq n$ and assume that $\widehat{\sigma}\left[p_{j}\right]$ are generalized full terms for all $1 \leq j \leq n$, then $\widehat{\sigma}[p]=S^{n}\left(\sigma\left(f_{i}\right)\right.$, $\left.\widehat{\sigma}\left[p_{1}\right], \ldots, \widehat{\sigma}\left[p_{n}\right]\right)$ is also a generalized full term.

Lemma 12. $H y p_{G F}(n)$ forms a submonoid of the monoid $H_{y p_{G}}(n)$ of all generalized hypersubstitutions.

Proof. Since $\sigma_{\mathrm{id}}(f)=f\left(x_{1}, \ldots, x_{n}\right)$ is a generalized full term, the identity-generalized hypersubstitution is a generalized full hypersubstitution.

Assume that $\sigma_{1}, \sigma_{2} \in \operatorname{Hyp}_{\mathrm{GF}}(n)$. Since $\left(\sigma_{1}{ }^{\circ} \mathrm{GF}_{2} \sigma_{2}\right)(f)=$ $\widehat{\sigma}_{1}\left[\sigma_{2}(f)\right]$ and $\sigma_{1}(f), \sigma_{2}(f)$ are generalized full terms, then by Lemma 11 we have that $\left(\sigma_{1}{ }^{\circ} \mathrm{GF} \sigma_{2}\right)(f)$ is a generalized full term. Hence $\sigma_{1}{ }^{\circ}{ }_{\mathrm{GF}} \sigma_{2} \in \operatorname{Hyp}_{\mathrm{GF}}(n)$ and therefore $\operatorname{Hyp}_{\mathrm{GF}}(n)$ forms a submonoid of $\operatorname{Hyp}_{G}(n)$.

Definition 13. Let $\sigma$ be a generalized full hypersubstitution of type $(n)$, and then we define

$$
\operatorname{depth}(\sigma)=\max \{\operatorname{depth}(\sigma(f)) \mid f \in \Omega\} .
$$

Corollary 14. Let $p=f\left(p_{1}, \ldots, p_{n}\right) \in W_{(n)}^{G F}(X), \sigma \in H y p_{G F}(n)$. Then

$$
\operatorname{depth}(\widehat{\sigma}[p])=\operatorname{depth}(\sigma(f)) \operatorname{depth}(p) .
$$

Proof. We give a proof by induction on $\operatorname{depth}(p)$.

If $\operatorname{depth}(p)=1$, then $p=f\left(x_{s(1)}, \ldots, x_{s(n)}\right)$ where $s \in$ $P_{n}$ or $p=f\left(x_{s^{\prime}\left(j_{1}\right)}, \ldots, x_{s^{\prime}\left(j_{n}\right)}\right)$ where $j_{1}, \ldots, j_{n}$ are natural numbers greater than $n$ and $s^{\prime}$ is a permutation on $\left\{j_{1}, \ldots, j_{n}\right\}$.

If $p=f\left(x_{s(1)}, \ldots, x_{s(n)}\right)$, then

$$
\begin{aligned}
\operatorname{depth}(\widehat{\sigma}[p]) & =\operatorname{depth}\left(\widehat{\sigma}\left[f\left(x_{s(1)}, \ldots, x_{s(n)}\right)\right]\right) \\
& =\operatorname{depth}\left(\sigma(f)_{s}\right) \\
& =\operatorname{depth}(\sigma(f)) \\
& =\operatorname{depth}(\sigma(f)) \operatorname{depth}(p) .
\end{aligned}
$$

If $p=f\left(x_{s^{\prime}\left(j_{1}\right)}, \ldots, x_{s^{\prime}\left(j_{n}\right)}\right)$, then

$$
\begin{aligned}
& \operatorname{depth}(\widehat{\sigma}[p]) \\
&=\operatorname{depth}\left(\widehat{\sigma}\left[f\left(x_{s^{\prime}\left(j_{1}\right)}, \ldots, x_{s^{\prime}\left(j_{n}\right)}\right)\right]\right) \\
&=\operatorname{depth}\left(S^{n}\left(\sigma(f), x_{s^{\prime}\left(j_{1}\right)}, \ldots, x_{s^{\prime}\left(j_{n}\right)}\right)\right) \\
&=\max \left\{\operatorname{depth}\left(x_{s^{\prime}\left(j_{1}\right)}\right), \ldots, \operatorname{depth}\left(x_{s^{\prime}\left(j_{n}\right)}\right)\right\} \\
&+\operatorname{depth}(\sigma(f)) \\
&= 0+\operatorname{depth}(\sigma(f)) \\
&= \operatorname{depth}(\sigma(f)) \\
&= \operatorname{depth}(\sigma(f)) \operatorname{depth}(p) .
\end{aligned}
$$

Assume that the $\operatorname{depth}\left(\widehat{\sigma}\left[p_{k}\right]\right)=\operatorname{depth}(\sigma(f)) \operatorname{depth}\left(p_{k}\right)$ for all $1 \leq k \leq n$ and $p=f\left(p_{1}, \ldots, p_{n}\right)$. Then

$$
\begin{aligned}
& \operatorname{depth}(\widehat{\sigma}[p]) \\
&=\operatorname{depth}\left(S^{n}\left(\sigma(f), \widehat{\sigma}\left[p_{1}\right], \ldots, \widehat{\sigma}\left[p_{n}\right]\right)\right) \\
&=\operatorname{depth}(\sigma(f))+\max \left\{\operatorname{depth}\left(\widehat{\sigma}\left[p_{k}\right]\right) \mid 1 \leq k \leq n\right\} \\
&=\operatorname{depth}(\sigma(f)) \\
&+\max \left\{\operatorname{depth}(\sigma(f)) \operatorname{depth}\left(p_{k}\right) \mid 1 \leq k \leq n\right\} \\
&= \operatorname{depth}(\sigma(f)) \\
&+\operatorname{depth}(\sigma(f)) \max \left\{\operatorname{depth}\left(p_{k}\right) \mid 1 \leq k \leq n\right\} \\
&= \operatorname{depth}(\sigma(f))\left(1+\max \left\{\operatorname{depth}\left(p_{k}\right) \mid 1 \leq k \leq n\right\}\right) \\
&= \operatorname{depth}(\sigma(f)) \operatorname{depth}(p) .
\end{aligned}
$$

Corollary 15. The function depth ${ }^{G F}: H y p_{G F}(n) \rightarrow \mathbb{N}^{+}$with $\sigma \mapsto$ depth $(\sigma)$ is a homomorphism from the monoid $\left(H y p_{G F}(n) ;{ }_{G F}, \sigma_{i d}\right)$ onto the monoid $\left(\mathbb{N}^{+} ; \cdot, 1\right)$.

Proof. The mapping depth ${ }^{\mathrm{GF}}$ is well defined and surjective since for every $n \in \mathbb{N}^{+}$there exists a generalized full term 
$p$ with $\operatorname{depth}(p)=n$. Furthermore, we have depth ${ }^{\mathrm{GF}}\left(\sigma_{\text {id }}\right)=$ $\operatorname{depth}\left(\sigma_{\text {id }}(f)\right)=\operatorname{depth}\left(f\left(x_{1}, \ldots, x_{n}\right)\right)=1$ and

$$
\begin{aligned}
& \operatorname{depth}\left(\left(\sigma_{1}{ }^{\circ}{ }_{\mathrm{GF}} \sigma_{2}\right)(f)\right)=\operatorname{depth}\left(\widehat{\sigma}_{1}\left[\sigma_{2}(f)\right]\right) \\
& =\operatorname{depth}\left(\sigma_{1}(f)\right) \operatorname{depth}\left(\sigma_{2}(f)\right) \\
& =\operatorname{depth}^{\mathrm{GF}}\left(\sigma_{1}\right) \operatorname{depth}^{\mathrm{GF}}\left(\sigma_{2}\right) \text {. }
\end{aligned}
$$

Proposition 16. Let $\sigma \in H y p_{G F}(n)$. Then $\widehat{\sigma}$ is an endomorphism on the algebra $\left(W_{(n)}^{G F}(X) ; S^{n}\right)$.

Proof. Indeed $\widehat{\sigma}:\left(W_{(n)}^{\mathrm{GF}}(X) ; S^{n}\right) \rightarrow\left(W_{(n)}^{\mathrm{GF}}(X) ; S^{n}\right)$ is a function. Now we give a proof by induction on the complexity of a term $t_{0}$ that, for any $t_{1}, \ldots, t_{n} \in W_{(n)}^{\mathrm{GF}}(X)$,

$$
\widehat{\sigma}\left[S^{n}\left(t_{0}, t_{1}, \ldots, t_{n}\right)\right]=S^{n}\left(\widehat{\sigma}\left[t_{0}\right], \ldots, \widehat{\sigma}\left[t_{n}\right]\right) .
$$

First consider the case that $t_{0}=f\left(x_{s(1)}, \ldots, x_{s(n)}\right)$ where $i \in I$ and $s \in P_{n}$. Then

$$
\begin{aligned}
\widehat{\sigma}\left[S^{n}\right. & \left.\left(t_{0}, t_{1}, \ldots, t_{n}\right)\right] \\
& =\widehat{\sigma}\left[S^{n}\left(f\left(x_{s(1)}, \ldots, x_{s(n)}\right), t_{1}, \ldots, t_{n}\right)\right] \\
& =\widehat{\sigma}\left[f\left(t_{s(1)}, \ldots, t_{s(n)}\right)\right] \\
& =S^{n}\left(\sigma(f), \widehat{\sigma}\left[t_{s(1)}\right], \ldots, \widehat{\sigma}\left[t_{s(n)}\right]\right) \\
& =S^{n}\left(\sigma(f)_{s}, \widehat{\sigma}\left[t_{1}\right], \ldots, \widehat{\sigma}\left[t_{n}\right]\right) \\
& =S^{n}\left(\widehat{\sigma}\left[t_{0}\right], \widehat{\sigma}\left[t_{1}\right], \ldots, \widehat{\sigma}\left[t_{n}\right]\right) .
\end{aligned}
$$

If $t_{0}=f\left(x_{s^{\prime}\left(j_{1}\right)}, \ldots, x_{s^{\prime}\left(j_{n}\right)}\right)$ where $j_{1}, \ldots, j_{n}$ are natural numbers greater than $n$ and $s^{\prime}$ is a permutation on $\left\{j_{1}, \ldots, j_{n}\right\}$, then

$$
\begin{aligned}
\widehat{\sigma}\left[S^{n}(\right. & \left.\left.t_{0}, t_{1}, \ldots, t_{n}\right)\right] \\
= & \widehat{\sigma}\left[S^{n}\left(f\left(x_{s^{\prime}\left(j_{1}\right)}, \ldots, x_{s^{\prime}\left(j_{n}\right)}\right), t_{1}, \ldots, t_{n}\right)\right] \\
= & \widehat{\sigma}\left[f\left(x_{s^{\prime}\left(j_{1}\right)}, \ldots, x_{s^{\prime}\left(j_{n}\right)}\right)\right] \\
= & S^{n}\left(\sigma(f), x_{s^{\prime}\left(j_{1}\right)}, \ldots, x_{s^{\prime}\left(j_{n}\right)}\right) \\
= & S^{n}\left(\sigma(f), \widehat{\sigma}\left[S^{n}\left(x_{s^{\prime}\left(j_{1}\right)}, t_{1}, \ldots, t_{n}\right)\right], \ldots,\right. \\
& \left.\quad \widehat{\sigma}\left[S^{n}\left(x_{s^{\prime}\left(j_{n}\right)}, t_{1}, \ldots, t_{n}\right)\right]\right) \\
= & S^{n}\left(S^{n}\left(\sigma(f), x_{s^{\prime}\left(j_{1}\right)}, \ldots, x_{s^{\prime}\left(j_{n}\right)}\right), \widehat{\sigma}\left[t_{1}\right], \ldots, \widehat{\sigma}\left[t_{n}\right]\right) \\
= & S^{n}\left(\widehat{\sigma}\left[f\left(x_{0}\right], \widehat{\sigma}\left[t_{1}\right], \ldots, \widehat{\sigma}\left[t_{n}\right]\right) .\right.
\end{aligned}
$$

If $t_{0}=f\left(r_{1}, \ldots, r_{n}\right)$ where $r_{1}, \ldots, r_{n} \in W_{(n)}^{\mathrm{GF}}(X)$ and assume that the formula is satisfied for $r_{1}, \ldots, r_{n}$, then

$$
\begin{aligned}
\widehat{\sigma}\left[S^{n}\left(t_{0}, t_{1}, \ldots, t_{n}\right)\right] & \\
= & \widehat{\sigma}\left[S^{n}\left(f\left(r_{1}, \ldots, r_{n}\right), t_{1}, \ldots, t_{n}\right]\right) \\
= & \widehat{\sigma}\left[f\left(S^{n}\left(r_{1}, t_{1}, \ldots, t_{n}\right), \ldots, S^{n}\left(r_{n}, t_{1}, \ldots, t_{n}\right)\right)\right] \\
= & S^{n}\left(\sigma(f), \widehat{\sigma}\left[S^{n}\left(r_{1}, t_{1}, \ldots, t_{n}\right)\right], \ldots,\right. \\
& \left.\widehat{\sigma}\left[S^{n}\left(r_{n}, t_{1}, \ldots, t_{n}\right)\right]\right) \\
= & S^{n}\left(\sigma(f), S^{n}\left(\widehat{\sigma}\left[r_{1}\right], \widehat{\sigma}\left[t_{1}\right], \ldots, \widehat{\sigma}\left[t_{n}\right]\right), \ldots,\right. \\
= & S^{n}\left(S^{n}\left(\sigma(f), \widehat{\sigma}\left[r_{1}\right], \ldots, \widehat{\sigma}\left[r_{n}\right]\right), \widehat{\sigma}\left[t_{1}\right], \ldots, \widehat{\sigma}\left[t_{n}\right]\right) \\
= & S^{n}\left(\widehat{\sigma}\left[t_{0}\right], \widehat{\sigma}\left[t_{1}\right], \ldots, \widehat{\sigma}\left[t_{n}\right]\right) .
\end{aligned}
$$

Since the free algebra clone $\mathrm{GF}_{\mathrm{G}}(n)$ is generated by the set

$$
\begin{aligned}
& \left\{f\left(x_{s(1)}, \ldots, x_{s(n)}\right) s \in P_{n}\right\} \\
& \quad \cup\left\{f\left(x_{s^{\prime}\left(j_{1}\right)}, \ldots, x_{s^{\prime}\left(j_{n}\right)}\right) \mid j_{1}, \ldots, j_{n}>n, s^{\prime}\right.
\end{aligned}
$$

is a permutation on $\left.\left\{j_{1}, \ldots, j_{n}\right\}\right\}$,

therefore any mapping $\eta$ from $\mathscr{F}_{\mathrm{GS}(n)}$ into $W_{(n)}^{\mathrm{GF}}(X)$ can be uniquely extended to an endomorphism $\bar{\eta}$ of clone $_{\mathrm{GF}}(n)$. These mappings are called generalized full clone substitutions. We denote the set of all generalized full clone substitutions by Subst ${ }_{\mathrm{GFC}}$. The set Subst $\mathrm{GFC}_{\mathrm{GF}}$ with a binary operation $\odot$ is defined by $\eta_{1} \odot \eta_{2}:=\bar{\eta}_{1} \circ \eta_{2}$, where $\circ$ is the usual composition of functions, and, together with id $\mathscr{F}_{G S(n)}$, the identity mapping on $\mathscr{F}_{\mathrm{GS}(n)}$ is a monoid. Then we have the following proposition.

Proposition 17. The monoid $\left(H y p_{G F}(n) ;{ }_{G F}, \sigma_{i d}\right)$ can be embedded into the monoid (Subst $t_{G F C} ; \odot, i d_{\mathscr{F}_{G S}(n)}$ ).

Proof. Let $\sigma \in \operatorname{Hyp}_{\mathrm{GF}}(n)$. Then by Proposition 16, $\widehat{\sigma}$ is an endomorphism on the algebra clone $\mathrm{GF}_{\mathrm{GF}}(n)$. Since $\mathscr{F}_{\mathrm{GS}(n)}=$ $\left\{f\left(x_{s(1)}, \ldots, x_{s(n)}\right) \mid s \in P_{n}\right\} \cup\left\{f\left(x_{s^{\prime}\left(j_{1}\right)}, \ldots, x_{s^{\prime}\left(j_{n}\right)}\right) \mid j_{1}, \ldots\right.$, $j_{n}>n, s^{\prime}$ is a permutation on $\left.\left\{j_{1}, \ldots, j_{n}\right\}\right\}$ is a generating set of clone $\mathrm{GF}_{\mathrm{GF}}(n)$, the mapping $\widehat{\sigma}_{\left.\right|_{\mathscr{F}_{\mathrm{GS}}(n)}}$ is a substitution with $\overline{\widehat{\sigma}_{I_{F_{\mathrm{GS}}(n)}}}=\widehat{\sigma}$. We define the mapping $\psi: \operatorname{Hyp}_{\mathrm{GF}}(n) \rightarrow$ Subst $\mathrm{GFC}_{\mathrm{GF}}$ by $\psi(\sigma)=\widehat{\sigma}_{\mathscr{I}_{\mathrm{GS}(n)}}$. Injectivity of $\psi$ is clear. We show that $\psi$ is a homomorphism. Let $\sigma_{1}, \sigma_{2} \in \operatorname{Hyp}_{\mathrm{GF}}(n)$. Then $\psi\left(\sigma_{1}{ }^{\circ}{ }_{G F} \sigma_{2}\right)=\left(\widehat{\sigma}_{1} \circ \sigma_{2}\right)_{\Upsilon_{\mathscr{F}_{\mathrm{GS}}(n)}}=\left(\widehat{\sigma}_{1} \circ \widehat{\sigma}_{2}\right)_{\left.\right|_{\mathscr{F}_{\mathrm{GS} S}(n)}}=\widehat{\sigma}_{1} \circ \widehat{\sigma}_{2_{\mid F_{\mathrm{GS}}(n)}}=$

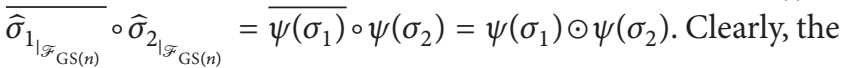
mapping $\psi$ preserves the identity element. 


\section{Full Strong Hyperidentities and Identities in clone $_{\mathrm{GF}}(n)$}

Let $V$ be the variety of type $(n)$ and let $\mathrm{Id}^{\mathrm{GF}} V:=W_{(n)}^{\mathrm{GF}}(X)^{2} \cap$ $\operatorname{Id} V$ be the set of all identities of $V$ consisting of generalized full terms. Then we have the following.

Proposition 18. $I d^{G F} V$ is a congruence on clone $_{G F}(n)$.

Proof. We will prove that if $r \approx t, r_{k} \approx t_{k} \in \mathrm{Id}^{\mathrm{GF}} V, k=$ $1,2, \ldots, n$, then $S^{n}\left(r, r_{1}, \ldots, r_{n}\right) \approx S^{n}\left(t, t_{1}, \ldots, t_{n}\right) \in \operatorname{Id}^{\mathrm{GF}} V$. Firstly, we give a proof by induction on the complexity of a term $t \in W_{(n)}^{\mathrm{GF}}(X)$ that, for every $n \in \mathbb{N}^{+}$from $t_{k} \approx$ $r_{k} \in \mathrm{Id}^{\mathrm{GF}} V, k=1,2, \ldots, n$, there follows $S^{n}\left(t, t_{1}, \ldots, t_{n}\right) \approx$ $S^{n}\left(t, r_{1}, \ldots, r_{n}\right) \in \mathrm{Id}^{\mathrm{GF}} V$.

If $t=f\left(x_{s(1)}, \ldots, x_{s(n)}\right)$ and $s \in P_{n}$, then

$$
\begin{aligned}
S^{n}(f & \left.\left(x_{s(1)}, \ldots, x_{s(n)}\right), t_{1}, \ldots, t_{n}\right) \\
& =f\left(t_{s(1)}, \ldots, t_{s(n)}\right) \\
& \approx f\left(r_{s(1)}, \ldots, r_{s(n)}\right) \\
& =S^{n}\left(f\left(x_{s(1)}, \ldots, x_{s(n)}\right), r_{1}, \ldots, r_{n}\right) \in \mathrm{Id}^{\mathrm{GF}} V,
\end{aligned}
$$

where $\operatorname{Id} V$ is compatible with the operation $\bar{f}$ of the absolutely free algebra $\mathscr{F}_{(n)}(X)$ and by the definition of generalized full terms.

If $t=f\left(x_{s^{\prime}\left(j_{1}\right)}, \ldots, x_{s^{\prime}\left(j_{n}\right)}\right)$ where $j_{1}, \ldots, j_{n}$ are natural numbers greater than $n$ and $s^{\prime}$ is a permutation on $\left\{j_{1}, \ldots, j_{n}\right\}$, then

$$
\begin{aligned}
S^{n}(f & \left.\left(x_{s^{\prime}\left(j_{1}\right)}, \ldots, x_{s^{\prime}\left(j_{n}\right)}\right), t_{1}, \ldots, t_{n}\right) \\
& =f\left(x_{s^{\prime}\left(j_{1}\right)}, \ldots, x_{s^{\prime}\left(j_{n}\right)}\right) \\
& =S^{n}\left(f\left(x_{s^{\prime}\left(j_{1}\right)}, \ldots, x_{s^{\prime}\left(j_{n}\right)}\right), r_{1}, \ldots, r_{n}\right) \in \mathrm{Id}^{\mathrm{GF}} V .
\end{aligned}
$$

Assume now that $t=f\left(l_{1}, \ldots, l_{n}\right) \in W_{(n)}^{\mathrm{GF}}(X)$ and, for $l_{j}, 1 \leq$ $j \leq n$, we have already

$$
S^{n}\left(l_{j}, t_{1}, \ldots, t_{n}\right) \approx S^{n}\left(l_{j}, r_{1}, \ldots, r_{n}\right) \in \mathrm{Id}^{\mathrm{GF}} V .
$$

Then

$$
\begin{aligned}
S^{n}(f & \left.\left(l_{1}, \ldots, l_{n}\right), t_{1}, \ldots, t_{n}\right) \\
& =f\left(S^{n}\left(l_{1}, t_{1}, \ldots, t_{n}\right), \ldots, S^{n}\left(l_{n}, t_{1}, \ldots, t_{n}\right)\right) \\
& \approx f\left(S^{n}\left(l_{1}, r_{1}, \ldots, r_{n}\right), \ldots, S^{n}\left(l_{n}, r_{1}, \ldots, r_{n}\right)\right) \\
& =S^{n}\left(f\left(l_{1}, \ldots, l_{n}\right), r_{1}, \ldots, r_{n}\right) \in \mathrm{Id}^{\mathrm{GF}} V .
\end{aligned}
$$

Now we prove the implication

$$
\begin{aligned}
t & \approx r \in \mathrm{Id}^{\mathrm{GF}} \mathrm{V} \Longrightarrow S^{n}\left(t, r_{1}, \ldots, r_{n}\right) \\
& \approx S^{n}\left(r, r_{1}, \ldots, r_{n}\right) \in \mathrm{Id}^{\mathrm{GF}} V .
\end{aligned}
$$

This is a consequence of the fully invariance of $\operatorname{Id} V$ as a congruence on the absolutely free algebra $\mathscr{F}_{(n)}(X)$ and the definition of a generalized full term. Assume now that $t \approx$ $r, t_{k} \approx r_{k} \in \mathrm{Id}^{\mathrm{GF}} V$. Then

$$
\begin{aligned}
S^{n}\left(t, t_{1}, \ldots, t_{n}\right) & \approx S^{n}\left(r, t_{1}, \ldots, t_{n}\right) \\
& \approx S^{n}\left(r, r_{1}, \ldots, r_{n}\right) \in \mathrm{Id}^{\mathrm{GF}} V .
\end{aligned}
$$

Definition 19. Let $V$ be a variety of type $(n)$ and let $\operatorname{Id}^{\mathrm{GF}} V$ be the set of all identities of $V$ consisting of generalized full terms. Then $s \approx t \in \mathrm{Id}^{\mathrm{GF}} V$ is called an $\Omega$-GF-hyperidentity in $V$ if $\widehat{\sigma}[s] \approx \widehat{\sigma}[t] \in \operatorname{Id}^{\mathrm{GF}} V$ for every $\sigma \in \operatorname{Hyp}_{\mathrm{GF}}(n)$. If every identity in $\mathrm{Id}^{\mathrm{GF}} V$ is satisfied as an $\Omega$-GF-hyperidentity, the variety $V$ is called $\Omega$-GF-solid.

Proposition 20. If $I d^{G F} V$ is a fully invariant congruence relation on clone ${ }_{G F}(n)$, then the variety $V$ is $\Omega-G F$-solid.

Proof. Let $s \approx t \in \operatorname{Id}^{\mathrm{GF}} V$ and let $\sigma \in \operatorname{Hyp}_{\mathrm{GF}}(n)$ be a generalized full hypersubstitution. Since by Proposition 16 the extension $\widehat{\sigma}$ of $\sigma$ is an endomorphism of clone $_{\mathrm{GF}}(n)$, we have $\widehat{\sigma}[s] \approx \widehat{\sigma}[t] \in \operatorname{Id}^{\mathrm{GF}} V$.

By Proposition 18 we can form a quotient algebra:

$$
\text { clone }_{\mathrm{GF}} V:=\text { clone }_{\mathrm{GF}}(n) / \mathrm{Id}^{\mathrm{GF}} V
$$

which belongs to a variety of a Menger algebra of rank $n$. There is the following connection between clone identities and $\Omega$-GF-hyperidentities in $V$.

Proposition 21. Let $V$ be the variety of type $(n)$ and let $s \approx$ $t \in I d^{G F} V$. If $s \approx t$ is an identity in clone $_{G F}(n)$, then it is an $\Omega$-GF-hyperidentity in $V$.

Proof. Let $s \approx t \in \mathrm{Id}^{\mathrm{GF}} V$ be an identity in clone $\mathrm{GF}_{\mathrm{GF}}(n)$ and let $\sigma \in \operatorname{Hyp}_{\mathrm{GF}}(n)$. Then $\widehat{\sigma} \in \operatorname{End}\left(\right.$ clone $\left._{\mathrm{GF}} \tau\right)$ the set of all endomorphisms of clone ${ }_{\mathrm{GF}}(n)$ and $\widehat{\sigma}_{\mathscr{F}_{\mathrm{GS}(\mathrm{n})}} \in$ Subst $_{\mathrm{GFC}}$ with $\overline{\widehat{\sigma}_{\mathscr{F}_{\mathrm{GS}(n)}}}=\widehat{\sigma}$. By the natural mapping natId ${ }^{\mathrm{GF}} V$ we have

$$
\operatorname{natId}^{\mathrm{GF}} V \circ \widehat{\sigma}_{\mathscr{F}_{\mathrm{GS}(n)}}: \mathscr{F}_{\mathrm{GS}(n)} \longrightarrow \text { clone }_{\mathrm{GF}}(n),
$$

and this is a valuation mapping with

$$
\overline{\text { natId }^{\mathrm{GF}} V \circ \widehat{\sigma}_{\mathscr{F}_{\mathrm{GS}(n)}}}=\operatorname{natId}^{\mathrm{GF}} V \circ \widehat{\sigma} .
$$

Then

$$
\begin{aligned}
s \approx & t \in \operatorname{Id}\left(\text { clone }_{\mathrm{GF}} V\right) \\
& \Longrightarrow \overline{\operatorname{natId}^{\mathrm{GF}} V \circ \widehat{\sigma}_{\mathscr{F}_{\mathrm{GS}(n)}}}(s)=\overline{\operatorname{natId}^{\mathrm{GF}} V \circ \widehat{\sigma}_{\mathscr{F}_{\mathrm{GS}(n)}}}(t) \\
& \Longrightarrow \operatorname{natId}^{\mathrm{GF}} V \circ \widehat{\sigma}(s)=\operatorname{natId}^{\mathrm{GF}} V \circ \widehat{\sigma}(t) \\
& \Longrightarrow[\widehat{\sigma}[s]]_{\mathrm{Id}^{\mathrm{GF}} V}=[\widehat{\sigma}[t]]_{\mathrm{Id}}{ }^{\mathrm{GF}} V \\
& \Longrightarrow \widehat{\sigma}[s] \approx \widehat{\sigma}[t] \in \mathrm{Id}^{\mathrm{GF}} V .
\end{aligned}
$$

This means that $s \approx t$ is satisfied as an $\Omega$-GF-hyperidentity in $V$. 


\section{Acknowledgments}

This work was supported by the Higher Education Commission, and the authors were supported by CHE Ph. D. Scholarship and the Faculty of Science of Chiang Mai University, Thailand. The authors are grateful to four referees for thorough reports that have helped us to improve the paper.

\section{References}

[1] J. Aczél, "Proof of a theorem on distributive type hyperidentities," Algebra Universalis, vol. 1, no. 1, pp. 1-6, 1971.

[2] V. D. Belousov, "Systems of quasigroups with generalized identities," Uspekhi Matematicheskikh Nauk, vol. 20, no. 1, pp. 75-146, 1965.

[3] W. D. Neumann, "Mal'cev conditions, spectra and Kronecker product," Journal of the Australian Mathematical Society A, vol. 25, no. 1, pp. 103-117, 1978.

[4] W. Taylor, "Hyperidentities and hypervarieties," Aequationes Mathematicae, vol. 23, no. 1, pp. 30-49, 1981.

[5] K. Denecke, D. Lau, R. Pöschel, and D. Schweigert, "Hyperidentities, hyperequational classes and clone congruences," in Contributions to General Algebra, vol. 7, pp. 97-118, HölderPichler-Tempsky, Vienna, Austria, 1991.

[6] S. Leeratanavalee and K. Denecke, "Generalized hypersubstitutions and strongly solid varieties," in General Algebra and Applications, Proceedings of the " 59 th Workshop on General Algebra", "15 th Conference for Young Algebraists Potsdam 2000", pp. 135-145, Shaker, 2000.

[7] K. Denecke, J. Koppitz, and S. Shtrakov, "The depth of a hypersubstitution," Journal of Automata, Languages and Combinatorics, vol. 6, no. 3, pp. 253-262, 2001.

[8] K. Denecke and Th. Changphas, "Full hypersubstitutions and fully-solid varieties of semigroups," East-West Journal of Mathematics, vol. 4, no. 1, pp. 101-112, 2002.

[9] A. Kelarev, Graph Algebras and Automata, Marcel Dekker, New York, NY, USA, 2003.

[10] A. V. Kelarev, Ring Constructions and Applications, World Scientific, River Edge, NJ, USA, 2002.

[11] J. Koppitz and K. Denecke, M-Solid Varieties of Algebras, Springer Science+Business Media, New York, NY, USA, 2006. 


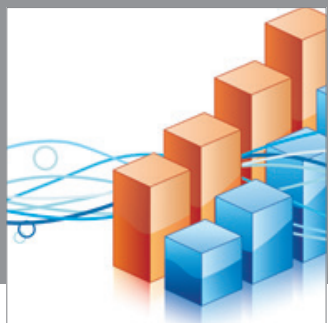

Advances in

Operations Research

mansans

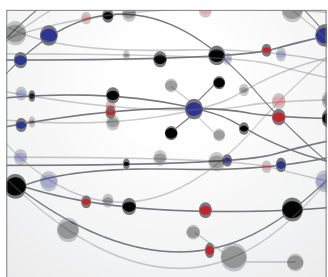

The Scientific World Journal
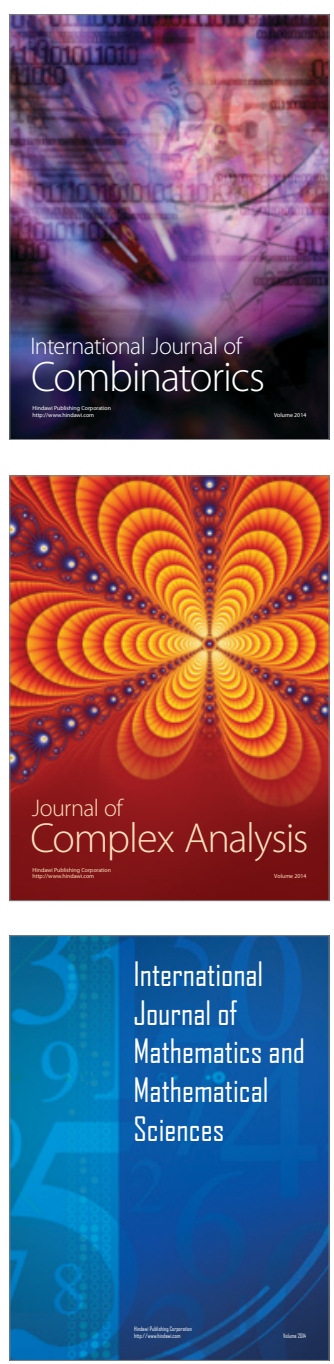
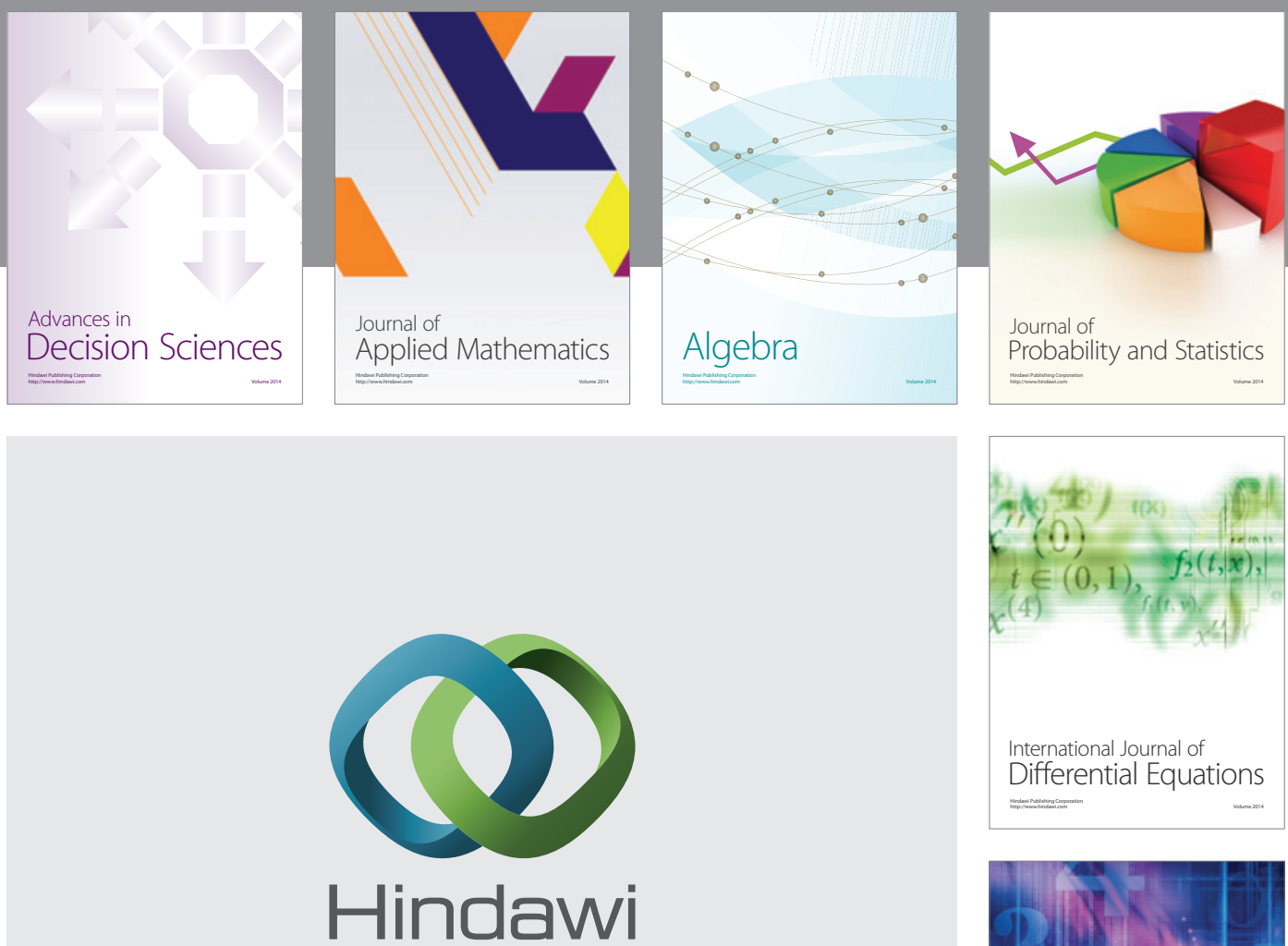

Submit your manuscripts at http://www.hindawi.com
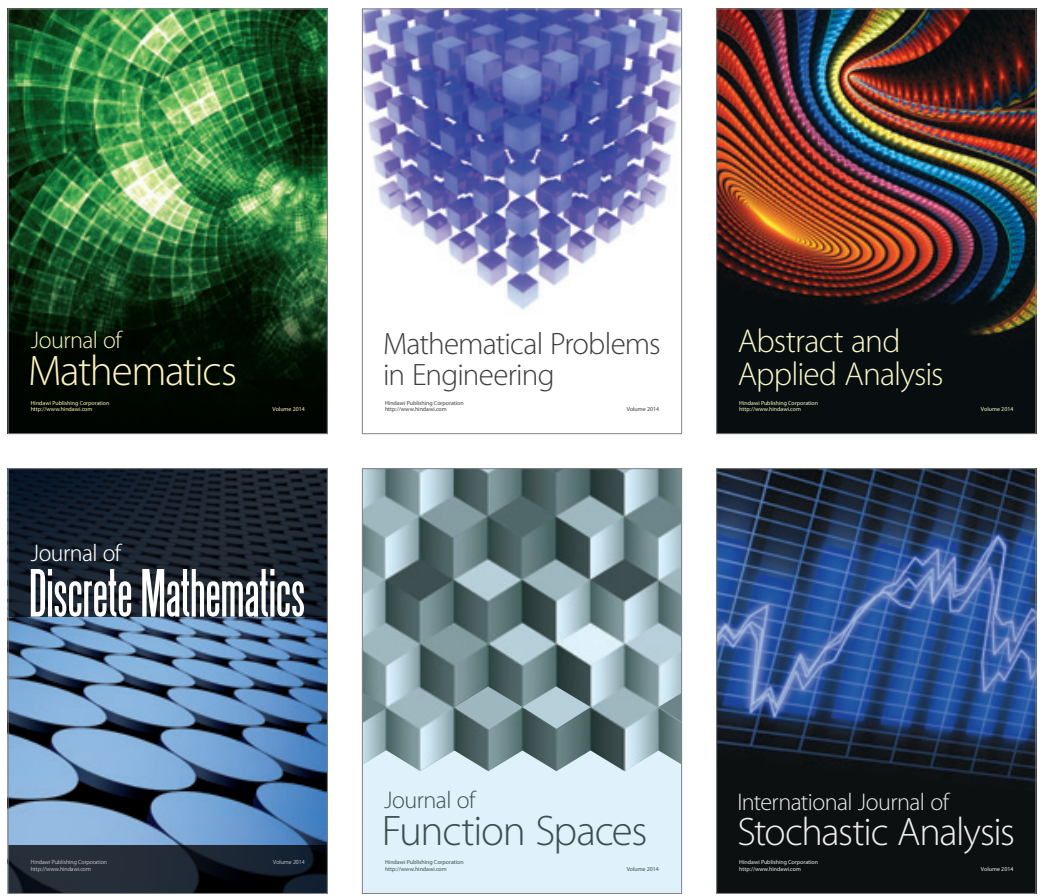

Journal of

Function Spaces

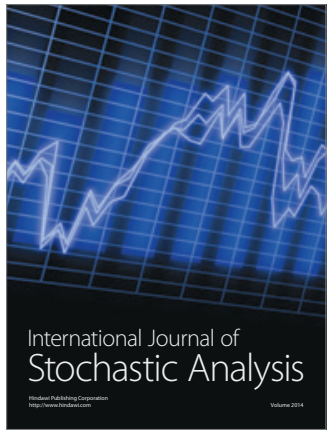

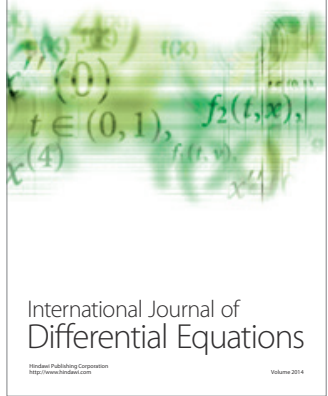
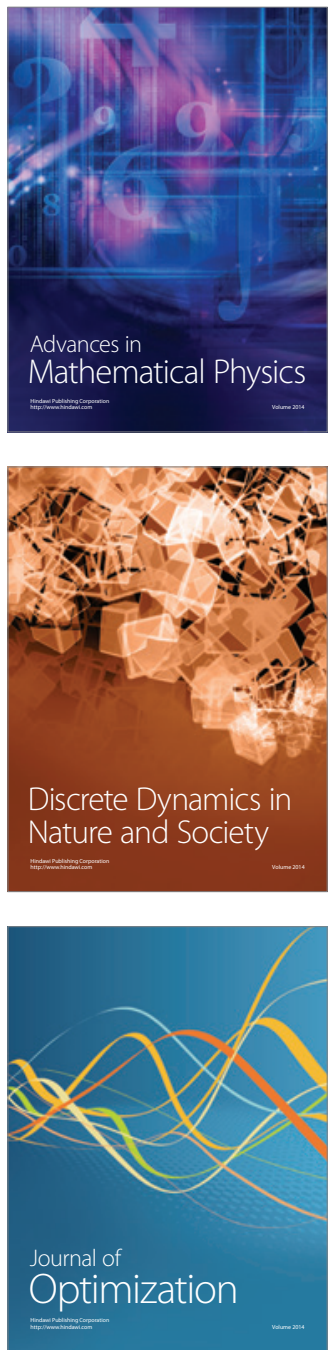